\title{
Acute kidney injury after pediatric liver transplantation: incidence, risk factors, and association with outcome
}

\section{AUTHOR(S):}

Hamada, Miho; Matsukawa, Shino; Shimizu, Satoshi; Kai, Shinichi; Mizota, Toshiyuki

\section{CITATION:}

Hamada, Miho ...[et al]. Acute kidney injury after pediatric liver transplantation: incidence, risk factors, and association with outcome. Journal of Anesthesia 2017, 31(5): 758-763

\section{ISSUE DATE:}

2017-10

URL:

http://hdl.handle.net/2433/234193

\section{RIGHT:}

This is a post-peer-review, pre-copyedit version of an article published in Journal of Anesthesia. The final authenticated version is available online at: http://dx.doi.org/10.1007/s00540-017-2395-2.; The full-text file will be made open to the public on 01 August 2018 in accordance with publisher's 'Terms and Conditions for Self-Archiving'.; この論文は出版社版 でありません。引用の際には出版社版をご確認ご利用ください。; This is not the published version. Please cite only the published version. 
Acute kidney injury after pediatric liver transplantation: incidence, risk factors, and association with outcome

Miho Hamada, MD, Shino Matsukawa, MD, Satoshi Shimizu, MD, PhD, Shinichi Kai, MD, PhD, Toshiyuki Mizota, MD, PhD

Department of Anesthesia, Kyoto University Hospital, 54 Shogoin-Kawahara-Cho, SakyoKu, Kyoto 606-8507, Japan

\section{Corresponding author:}

Toshiyuki Mizota, MD, PhD

Department of Anesthesia, Kyoto University Hospital, 54 Shogoin-Kawahara-Cho, SakyoKu, Kyoto 606-8507, Japan

Tel: +81-75-751-3433, Fax: +81-75-752-3259

E-mail: mizota@kuhp.kyoto-u.ac.jp

Key words: acute kidney injury, adolescent, child, infant, liver transplantation

Word count excluding abstract and references: 2,269

Number of tables: 2

Number of figures: 2 


\begin{abstract}
Purpose: Data on the incidence of, risk factors for, and association with outcomes of acute kidney injury (AKI) after pediatric liver transplantation are scarce. We conducted a retrospective cohort study to determine the incidence of AKI after pediatric liver transplantation. In addition, we examined risk factors for AKI and association of AKI with outcomes.
\end{abstract}

Methods: This study included 156 children aged between 3 months and 18 years undergoing liver transplantation at Kyoto University Hospital. AKI was defined according to the Kidney Disease: Improving Global Outcomes guidelines based on serum creatinine and urine output. We used multivariable logistic regression with stepwise variable selection to identify independent risk factors for AKI.

Results: AKI occurred in 72 patients (46.2\%); 34 (21.8\%) had Stage 1, 32 (20.5\%) had Stage 2, and 6 (3.8\%) had Stage 3 AKI. Factors independently associated with the development of AKI were increased preoperative total bilirubin level (adjusted odds ratio: 1.04 per $1 \mathrm{mg} / \mathrm{dL}$; 95\% confidence interval: 1.01-1.09; $\mathrm{P}=0.026$ ) and increased intraoperative blood loss (adjusted odds ratio: 1.03 per $10 \mathrm{~mL} / \mathrm{kg}$; 95\% confidence interval: 1.00-1.06; $\mathrm{P}=0.022$ ). AKI was significantly associated with prolonged hospitalization (median: 61 vs. 46 days; $\mathrm{P}=$ 0.028). In-hospital mortality rate was $4.2 \%$ in patients with AKI and $3.6 \%$ in those without (P $=1.000)$.

Conclusion: The incidence of AKI after pediatric liver transplantation was $46.2 \%$. Increased preoperative total bilirubin level and increased intraoperative blood loss were independently associated with the development of AKI. AKI was associated with prolonged hospitalization. 


\section{Introduction}

Previous studies involving adults have shown that acute kidney injury (AKI) is common after liver transplantation and may lead to serious complications including the development of chronic kidney disease and increased mortality [1-3]. A single-center study involving 424 adult liver transplant recipients [3] used the Kidney Disease: Improving Global Outcomes criteria to define postoperative AKI and reported that AKI occurred in 52\% of patients and the development of AKI negatively impacted short-term and long-term graft survival. Many patient- and procedure-related risk factors for AKI after adult liver transplantation have been identified, including female gender [3], obesity [3,4], preoperative chronic kidney disease [5,6], diabetes mellitus [1,7,8], high model for end-stage liver disease score [4,8-12], high Child-Pugh score [3], and large amount of blood loss [6,9,13]. These findings may help identify patients at a high risk for AKI and improve perioperative management to prevent or ameliorate AKI.

However, in contrast to adult liver transplantation, data on the incidence of, risk factors for, and association with outcomes of AKI in pediatric liver transplant recipients are scarce. Because adults and children differ in terms of the etiology of liver disease, coexisting conditions, and reserve capacity of the kidney, the epidemiologic features of AKI after pediatric liver transplantation may differ from those after adult liver transplantation. Therefore, we conducted a retrospective cohort study to determine the incidence of AKI after pediatric liver transplantation. We hypothesized that AKI would be common among pediatric liver transplant recipients. In addition, we investigated the risk factors for AKI and association of AKI with outcomes. 


\section{Methods}

The ethics committee of Kyoto University Hospital approved this single-center retrospective study (approval number: R0060; April 14, 2016) and waived the requirement for informed consent. We included patients aged between 3 months and 18 years who underwent liver transplantation at Kyoto University Hospital from January 2006 to March 2015. Patients who had end-stage renal disease preoperatively (patients having an estimated glomerular filtration rate of $<35 \mathrm{~mL} / \mathrm{min} / 1.73 \mathrm{~m}^{2}$, as determined using the modified Schwartz equation [14], or those undergoing hemodialysis within 2 weeks prior to the surgery) were excluded because AKI assessment was no longer relevant.

\section{Perioperative management}

At our institution, anesthetic drugs used for liver transplantation are standardized; anesthesia was induced using propofol (1-1.5 mg/kg) and remifentanil (0.1 $\mu \mathrm{g} / \mathrm{kg} / \mathrm{min})$, and maintained using sevoflurane (1\%-1.5\%) and remifentanil (0.1-0.3 $\mu \mathrm{g} / \mathrm{kg} / \mathrm{min})$. During the surgery, red blood cell concentrate was administered to maintain a hematocrit of 25\%-30\%. Although use of vasopressors was decided by the attending anesthesiologist, the first-line vasopressor used during the surgery was phenylephrine. If phenylephrine was ineffective, dopamine, norepinephrine, vasopressin, or epinephrine was considered.

Immunosuppression was provided according to the institutional protocol, as previously described [15]. The basic immunosuppressive regimen comprised a calcineurin inhibitor (tacrolimus or cyclosporine) and low-dose corticosteroid. Additionally, recipients of bloodtype-incompatible liver grafts received preoperative anti-CD20 antibody (rituximab, 375 $\mathrm{mg} / \mathrm{m}^{2}$ ) with preoperative plasma exchange, postoperative hepatic artery infusion of prostaglandin E1 (0.01 $\mu \mathrm{g} / \mathrm{kg} / \mathrm{min})$ and methylprednisolone (125 mg/day), and postoperative 
cyclophosphamide (2 mg/kg/day), followed by mycophenolate mofetil (starting dose, 500 mg/day; maintenance dose, 1000 mg/day).

\section{Data collection}

The following patient and perioperative variables were obtained from patients’ medical records: patient characteristics (age, gender, height, weight, etiology of liver disease, preoperative laboratory test results, and Child-Pugh classification), operative variables (retransplantation, intraoperative blood loss, graft-to-recipient weight ratio, blood-type compatibility, and cold and warm ischemia times), and postoperative course [urine output, laboratory test results, postoperative hemodialysis, durations of postoperative intensive care unit (ICU) and hospital stays, and in-hospital mortality].

\section{Outcomes}

The primary outcome was AKI defined as any stage of AKI according to the Kidney Disease: Improving Global Outcomes guidelines [16]. The AKI stages were based on the serum creatinine (SCr) concentration measured daily for 7 days after surgery and urine output measured every $2 \mathrm{~h}$ during the postoperative ICU stay (for 7 days after surgery if the postoperative ICU stay continues longer than 7 days). The most recent SCr level measured before the surgery was used as the baseline value.

\section{Statistical analyses}

Continuous variables were presented as medians (interquartile range) and compared using the Mann-Whitney U test; categorical variables were presented as numbers (percentage) and compared using the Pearson chi-square test or Fisher exact test, as appropriate. We applied multivariable logistic regression analysis to determine the independent predictors of 
postoperative AKI. On the basis of clinical relevance and a literature search for factors shown to be associated with AKI after adult liver transplantation, 12 variables [age, gender, preoperative laboratory test results (estimated glomerular filtration rate, serum albumin, prothrombin activity, and total bilirubin), hepatic encephalopathy, ascites, re-transplantation, intraoperative blood loss, graft-to-recipient weight ratio, and warm ischemia time] were considered for entry into the model [3-13]. Multicollinearity among variables was assessed by the variance inflation factor with a reference value of 10 . A stepwise method combined with a bootstrap procedure [17] was used for variable selection. After considering all variables, a stepwise (forward and backward) variable selection minimizing Akaike information criteria was repeated for each of the 1000 bootstrap resamplings, and variables selected in $50 \%$ or more of the bootstrap models were retained in the final model. The discrimination and the calibration of the model were assessed using the c-statistic and the Hosmer-Lemeshow goodness-of-fit test, respectively. The primary multivariable analysis was based on data from patients for whom complete information on all variables was available. Sensitivity analyses were performed with the use of multiple imputation by chained equations. Missing values were imputed conditional on the non-missing values of the other variables including age, gender, estimated glomerular filtration rate, serum albumin, prothrombin activity, total bilirubin, hepatic encephalopathy, ascites, re-transplantation, intraoperative blood loss, graft-to-recipient weight ratio, warm ischemia time, and the presence or absence of AKI. For each outcome, multiple imputation results were obtained from 100 imputed datasets.

Time-to-event analyses were used to compare the lengths of ICU and hospital stays. Patient data were censored at the time of death. Medians and interquartile ranges were obtained using Kaplan-Meier analyses, and the log-rank test was used to assess differences between groups. The sample size was determined by including all cases during the study period to maximize 
the power. Previous studies suggested that at least 8-10 events per variable were required for reliable multivariable logistic regression analysis [18,19]. We assumed 20 eligible surgeries per year and predicted the prevalence of AKI to be 33\%-78\% on the basis of published reports concerning adult liver transplantation. Thus, we estimated that we could conduct multivariable logistic regression with 6-14 variables using our dataset.

All statistical tests were two tailed, and a P value of $<0.05$ was considered to be statistically significant. All statistical analyses were performed using the statistical program $\mathrm{R}$ (http://cran.r-project.org). 


\section{Results}

A total of 165 pediatric patients underwent liver transplantation during the study period. Of these, 156 subjects were analyzed, after excluding eight patients who had preoperative endstage renal disease and one patient whose data on postoperative urine output were missing and, therefore, for whom the presence or absence of AKI could not be judged. Only one case of cadaveric liver transplantation was included; all others were living donor liver transplantations. The median age of the study participants was 2 years (interquartile range: 0 6); 90 patients (57.7\%) were female. AKI occurred in 72 patients (46.2\%); 34 (21.8\%) had Stage 1, 32 (20.5\%) had Stage 2, and 6 (3.8\%) had Stage 3 AKI. AKI defined according to the SCr level alone developed in 53 patients (34.0\%), and AKI defined according to urine output alone developed in 35 patients (22.4\%). In 19 of the 35 patients (54.3\%) who met the urine output criterion for AKI, the diagnosis of AKI would have been missed if only the SCr criterion had been used (Figure 1).

Univariable associations between perioperative characteristics and postoperative AKI are presented in Table 1. Variables significantly associated at the 5\% level were preoperative prothrombin activity, preoperative total bilirubin, and intraoperative blood loss.

One hundred forty-three patients were included in the multivariable logistic regression analysis after excluding 13 patients (8.3\%) with missing variables. After stepwise variable selection with bootstrap resampling, preoperative total bilirubin and intraoperative blood loss were chosen in more than $50 \%$ of resamples (preoperative total bilirubin: $72.6 \%$; intraoperative blood loss: 57.0\%) and included in the final model. In the final multivariable model, increased preoperative total bilirubin level and increased intraoperative blood loss were independently associated with the development of AKI (Table 2). None of the variance inflation factor values were greater than 10 , indicating that there was no multicollinearity. The final model had acceptable discrimination (c-statistic: 0.651; 95\% confidence interval: 
0.559-0.742) and calibration (Hosmer-Lemeshow statistic P value: 0.472). When using multiple imputation to account for missing values, the point estimates for the associations were not substantially affected; the adjusted odds ratio for total bilirubin was 1.04 (95\% confidence interval: $1.01-1.08 ; \mathrm{P}=0.024)$ and that for intraoperative blood loss was 1.02 (95\% confidence interval: 0.99-1.06; $\mathrm{P}=0.108$ ).

We assessed the association of AKI with the lengths of ICU and hospital stays and in-hospital mortality. There was no significant difference in ICU stay between patients with and without AKI [median (interquartile range): 4 (4-5) vs. 4 (4-5) days; P = 0.094; Figure 2A], whereas AKI was significantly associated with prolonged hospitalization [median (interquartile range): 61 (44-100) vs. 46 (35-78) days; $\mathrm{P}=0.028$; Figure 2B]. We could not find a significant association between AKI and in-hospital mortality (4.2\% in patients with AKI and 3.6\% in those without; $\mathrm{P}=1.000$ ). Clinical outcomes stratified by AKI stages or AKI definitions are presented in Supplemental data 1. 


\section{Discussion}

In this retrospective study of 156 children aged between 3 months and 18 years who underwent liver transplantation, we found that the incidence of postoperative AKI according to recent guidelines was $46.2 \%$. In addition, increased preoperative total bilirubin concentration and increased intraoperative blood loss were independently associated with the development of AKI. AKI was significantly associated with a prolonged hospital stay. To the best of our knowledge, this is the first study to investigate the incidence of postoperative AKI after pediatric liver transplantation. Children are assumed to have a low prevalence of chronic diseases such as diabetes mellitus and chronic kidney disease, both of which have been shown to be risk factors for AKI after liver transplantation [1,5-8]. In our study, only one patient had diabetes mellitus and no patient had preoperative renal insufficiency with an estimated glomerular filtration rate of $<60 \mathrm{~mL} / \mathrm{min} / 1.73 \mathrm{~m}^{2}$. Despite these low prevalences of risk factors, the incidence of AKI in the current study (46.2\%) was similar to that reported in adult liver transplantation $(39.4 \%-56.6 \%)[3,20,21]$. The common occurrence of AKI reinforces the need for systematic surveillance for AKI after pediatric liver transplantation.

Compared with applying the SCr criterion alone, incorporating oliguria into the diagnostic criteria increased the measured incidence of AKI from 34.0\% to 46.2\%. Assessment of AKI according to the SCr level alone failed to identify AKI in 54.3\% of the patients with low urine output. These findings are similar to our previous study in adult liver transplantation patients wherein we demonstrated that $44.7 \%$ of patients with oliguria were missed when we diagnosed AKI using the SCr level alone [15]. Epidemiological study of AKI in critically ill children and young adults showed that low urine output without increased SCr conferred an increased risk for mortality [22]. Our findings reveal the importance of monitoring both the SCr level and the urine output. 
One of the risk factors for AKI identified in our study was increased preoperative total bilirubin level. This finding is in line with previous studies of AKI in adult liver transplant recipients, reporting that a high model for end-stage liver disease score or Child-Pugh score (both are calculated using the total bilirubin level) were independent risk factors for AKI [3,4,8-12]. Increased preoperative total bilirubin might reflect the severity of the underlying liver disease. Intraoperative blood loss was also found to be an independent risk factor for postoperative AKI in our study, as was shown in previous studies in adult liver transplant recipients $[6,9,13]$. Blood loss appears to be a surrogate marker for surgical complexity or intraoperative adverse events, and hemodynamic instability or anemia due to massive bleeding might reduce oxygen delivery to the kidneys and cause kidney injury.

Prolonged hospitalization of patients with AKI might suggest that the postoperative course of these patients is complicated, although this finding is merely an association and cannot imply a causal relationship. Because in-hospital mortality in the study participants was very low (3.8\%), this study is underpowered to assess differences in mortality by AKI status. However, AKI was shown to be associated with poor outcomes including increased mortality in critically ill children [22] and children undergoing cardiac surgery [23-26].

Limitations of our study are as follows. This was a single-center study, which may reduce the generalizability of our results. Most patients in this study underwent living donor liver transplantation; we cannot confirm whether our findings can be extrapolated to pediatric patients undergoing cadaveric liver transplantation. We used urine output measured every $2 \mathrm{~h}$ instead of every hour to define AKI, because urine output measurement is performed every 2 $\mathrm{h}$ at our ICU; this modification of the definition of AKI might have affected the incidence of AKI.

In conclusion, the incidence of postoperative AKI after pediatric liver transplantation was 46.2\%. Increased preoperative total bilirubin level and increased intraoperative blood loss 
were independently associated with the development of AKI. AKI was significantly associated with a prolonged hospital stay. 


\section{Acknowledgments}

This work was supported in part by the JSPS KAKENHI (grant number 16K20092; TM, principle investigator). 


\section{References}

1. Barri YM, Sanchez EQ, Jennings LW, Melton LB, Hays S, Levy MF, Klintmalm GB. Acute kidney injury following liver transplantation: definition and outcome. Liver Transpl. 2009;15:475-83.

2. Ferreira AC, Nolasco F, Carvalho D, Sampaio S, Baptista A, Pessegueiro P, Monteiro E, Mourão L, Barroso E. Impact of RIFLE classification in liver transplantation. Clin Transplant. 2010;24:394-400.

3. Hilmi IA, Damian D, Al-Khafaji A, Planinsic R, Boucek C, Sakai T, Chang CC, Kellum JA. Acute kidney injury following orthotopic liver transplantation: incidence, risk factors, and effects on patient and graft outcomes. Br J Anaesth. 2015;114:919-26.

4. Park MH, Shim HS, Kim WH, Kim HJ, Kim DJ, Lee SH, Kim CS, Gwak MS, Kim GS. Clinical Risk Scoring Models for Prediction of Acute Kidney Injury after Living Donor Liver Transplantation: A Retrospective Observational Study. PLoS One. 2015;10:e0136230.

5. Bilbao I, Charco R, Balsells J, Lazaro JL, Hidalgo E, Llopart L, Murio E, Margarit C. Risk factors for acute renal failure requiring dialysis after liver transplantation. Clin Transplant. 1998;12:123-9.

6. Lebrón Gallardo M, Herrera Gutierrez ME, Seller Pérez G, Curiel Balsera E, Fernández Ortega JF, Quesada García G. Risk factors for renal dysfunction in the postoperative course of liver transplant. Liver Transpl. 2004;10:1379-85.

7. Pawarode A, Fine DM, Thuluvath PJ. Independent risk factors and natural history of renal dysfunction in liver transplant recipients. Liver Transpl. 2003;9:741-7.

8. Utsumi M, Umeda Y, Sadamori H, Nagasaka T, Takaki A, Matsuda H, Shinoura S, Yoshida R, Nobuoka D, Satoh D, Fuji T, Yagi T, Fujiwara T. Risk factors for acute renal injury in living donor liver transplantation: evaluation of the RIFLE criteria. Transpl Int. 2013;26:842-52. 
9. Lee SK, Park JB, Kim SJ, Choi GS, Kim DJ, Kwon CH, Lee SK, Joh JW. Early postoperative renal dysfunction in the adult living donor liver transplantation. Transplant Proc. 2007;39:1517-9.

10. Zhu M, Li Y, Xia Q, Wang S, Qiu Y, Che M, Dai H, Qian J, Ni Z, Axelsson J, Yan Y. Strong impact of acute kidney injury on survival after liver transplantation. Transplant Proc. 2010;42:3634-8.

11. Umbro I, Tinti F, Mordenti M, Rossi M, Ianni S, Pugliese F, Ruberto F, Ginanni

Corradini S, Nofroni I, Poli L, Berloco PB, Mitterhofer AP. Model for end-stage liver disease score versus simplified acute physiology score criteria in acute renal failure after liver transplantation. Transplant Proc. 2011;43:1139-41.

12. Romano TG, Schmidtbauer I, Silva FM, Pompilio CE, D'Albuquerque LA, Macedo E. Role of MELD score and serum creatinine as prognostic tools for the development of acute kidney injury after liver transplantation. PLoS One. 2013;8:e64089.

[13] Chen J, Singhapricha T, Hu KQ, Hong JC, Steadman RH, Busuttil RW, Xia VW. Postliver transplant acute renal injury and failure by the RIFLE criteria in patients with normal pretransplant serum creatinine concentrations: a matched study. Transplantation 2011;91:348-53.

14. Schwartz GJ, Brion LP, Spitzer A. The use of plasma creatinine concentration for estimating glomerular filtration rate in infants, children, and adolescents. Pediatr Clin North Am. 1987;34:571-90.

15. Mizota T, Minamisawa S, Imanaka Y, Fukuda K. Oliguria without serum creatinine increase after living donor liver transplantation is associated with adverse postoperative outcomes. Acta Anaesthesiol Scand. 2016;60:874-81. 
16. Kidney Disease: Improving Global Outcomes (KDIGO) Acute Kidney Injury Work Group. KDIGO clinical practice guidelines for acute kidney injury. Kidney Int Suppl. 2012;2:1-138.

17. Sauerbrei W, Schumacher M. A bootstrap resampling procedure for model building: application to the Cox regression model. Stat Med. 1992;11:2093-109.

18. Peduzzi P, Concato J, Kemper E, Holford TR, Feinstein AR. A simulation study of the number of events per variable in logistic regression analysis. J Clin Epidemiol. 1996;49:1373-9.

19. Cepeda MS, Boston R, Farrar JT, Strom BL. Comparison of logistic regression versus propensity score when the number of events is low and there are multiple confounders. Am J Epidemiol. 2003;158:280-7.

20. Klaus F, Keitel da Silva C, Meinerz G, Carvalho LM, Goldani JC, Cantisani G, Zanotelli ML, Duro Garcia V, Keitel E. Acute kidney injury after liver transplantation: incidence and mortality. Transplant Proc. 2014;46:1819-21.

21. Karapanagiotou A, Dimitriadis C, Papadopoulos S, Kydona C, Kefsenidis S, Papanikolaou V, Gritsi-Gerogianni N. Comparison of RIFLE and AKIN criteria in the evaluation of the frequency of acute kidney injury in post-liver transplantation patients. Transplant Proc. 2014;46:3222-7.

22. Kaddourah A, Basu RK, Bagshaw SM, Goldstein SL; AWARE Investigators. Epidemiology of Acute Kidney Injury in Critically Ill Children and Young Adults. N Engl J Med. 2017;376:11-20.

23. Aydin SI, Seiden HS, Blaufox AD, Parnell VA, Choudhury T, Punnoose A, Schneider J. Acute kidney injury after surgery for congenital heart disease. Ann Thorac Surg. 2012;94:1589-95. 
24. Tóth R, Breuer T, Cserép Z, Lex D, Fazekas L, Sápi E, Szatmári A, Gál J, Székely A. Acute kidney injury is associated with higher morbidity and resource utilization in pediatric patients undergoing heart surgery. Ann Thorac Surg. 2012;93:1984-90.

25. Ricci Z, Di Nardo M, Iacoella C, Netto R, Picca S, Cogo P. Pediatric RIFLE for acute kidney injury diagnosis and prognosis for children undergoing cardiac surgery: a singlecenter prospective observational study. Pediatr Cardiol. 2013;34:1404-8.

26. Gil-Ruiz Gil-Esparza MA, Alcaraz Romero AJ, Romero Otero A, Gil Villanueva N, Sanavia Morán E, Rodríguez Sánchez de la Blanca A, Lorente Romero J, Bellón Cano JM. Prognostic relevance of early AKI according to pRIFLE criteria in children undergoing cardiac surgery. Pediatr Nephrol. 2014;29:1265-72. 
Table 1. Univariable association between perioperative characteristics and postoperative AKI

\begin{tabular}{|c|c|c|c|c|}
\hline \multirow{2}{*}{ Variables } & \multirow{2}{*}{ All patients $(\mathrm{n}=156)$} & \multicolumn{2}{|c|}{ Acute kidney injury } & \multirow{2}{*}{$\mathrm{P}$ value } \\
\hline & & Absent $(\mathrm{n}=84)$ & Present $(\mathrm{n}=72)$ & \\
\hline Age (years) & $2(0-6)$ & $2(0-6)$ & $1(0-8)$ & 0.514 \\
\hline Female gender & 90 (57.7\%) & 48 (57.1\%) & $42(58.3 \%)$ & 1.000 \\
\hline Height (cm) & $81(66-113)$ & $85(67-110)$ & $72(65-118)$ & 0.402 \\
\hline Weight (kg) & $11(7-21)$ & $12(7-20)$ & $9(7-22)$ & 0.683 \\
\hline Etiology & & & & 0.888 \\
\hline Biliary atresia & $112(71.8 \%)$ & $60(71.4 \%)$ & $52(72.2 \%)$ & \\
\hline Metabolic liver disease & $9(5.8 \%)$ & $6(7.1 \%)$ & $3(4.2 \%)$ & \\
\hline Hepatic malignancy & $8(5.1 \%)$ & $5(6.0 \%)$ & $3(4.2 \%)$ & \\
\hline Acute liver failure & $2(1.3 \%)$ & $1(1.2 \%)$ & $1(1.4 \%)$ & \\
\hline Others & $25(16.0 \%)$ & $12(14.3 \%)$ & $13(18.1 \%)$ & \\
\hline Child-Pugh classification (A/B/C) & $30 / 72 / 54$ & $19 / 42 / 23$ & $11 / 30 / 31$ & 0.115 \\
\hline Ascites & 49 (31.4\%) & $23(27.4 \%)$ & $26(36.1 \%)$ & 0.300 \\
\hline Encephalopathy & $6(3.8 \%)$ & $2(2.4 \%)$ & $4(5.6 \%)$ & 0.416 \\
\hline
\end{tabular}




$\begin{array}{lcccc}\text { Preoperative serum creatinine (mg/dL) } & 0.20(0.14-0.30) & 0.20(0.20-0.30) & 0.20(0.10-0.27) & 0.070 \\ \text { Preoperative estimated glomerular } & & & & \\ \text { filtration rate (mL/min/1.73 m²) } & 230.7(163.9-286.6) & 221.8(159.8-273.2) & 242.0(175.3-293.1) & 0.121 \\ \text { Preoperative serum albumin (g/dL) } & 3.2(2.7-3.7) & 3.2(2.7-3.6) & 3.1(2.7-3.7) & 0.813 \\ \text { Preoperative prothrombin activity (\%) } & 65(51-83) & 69(53-89) & 63(43-73) & 0.017 \\ \text { Preoperative international normalized } & 1.31(1.11-1.59) & 1.25(1.06-1.52) & 1.37(1.22-1.70) & 0.008 \\ \text { ratio of prothrombin time } & 7.7(1.3-16.2) & 4.6(1.1-13.5) & 9.7(2.7-21.5) & 0.006 \\ \text { Preoperative total bilirubin (mg/dL) } & 15(9.6 \%) & 5(6.0 \%) & 10(13.9 \%) & 0.109 \\ \text { Re-transplantation } & 87.8(47.4-143.9) & 75.5(41.6-121.5) & 106.0(50.9-188.1) & 0.026 \\ \text { Blood loss (mL/kg) } & 2.47(1.43-3.43) & 2.43(1.43-3.36) & 2.65(1.55-3.43) & 0.976 \\ \text { Graft-to-recipient weight ratio (\%) } & 30(19.2 \%) & 19(22.6 \%) & 11(15.3 \%) \\ \text { ABO incompatible } & 85(54-138) & 80(54-126) & 91(55-156) & 0.309 \\ \text { Cold ischemia time (min) } & 38(33-44) & 39(35-45) & 0.202 \\ \text { Warm ischemia time (min) } & & & 0.158\end{array}$

Height was missing in 1 patient with AKI and 3 without AKI, and estimated glomerular filtration rate could not be calculated in these patients; blood loss was missing in 5 patients with AKI and 3 without AKI; graft-to-recipient weight ratio was missing in 4 patients with AKI and 3 without 
AKI; cold ischemia time was missing in 6 patients with AKI and 4 without AKI; warm ischemia time was missing in 6 patients with AKI and 3 without AKI. AKI, acute kidney injury. 
Table 2. Independent risk factors for acute kidney injury

\begin{tabular}{lcc}
\hline & Adjusted odds ratio (95\% confidence interval) & P value \\
\hline Preoperative total bilirubin (per 1 mg/dL) & $1.04(1.01-1.09)$ & 0.026 \\
Intraoperative blood loss (per $10 \mathrm{~mL} / \mathrm{kg})$ & $1.03(1.00-1.06)$ & 0.022 \\
\hline
\end{tabular}


Figure legends

Figure 1. Venn diagram of AKI status based on SCr or urine output criteria. AKI, acute kidney injury; SCr, serum creatinine.

\section{SCr criterion}

Non-oliguric AKI

$\mathrm{n}=37$

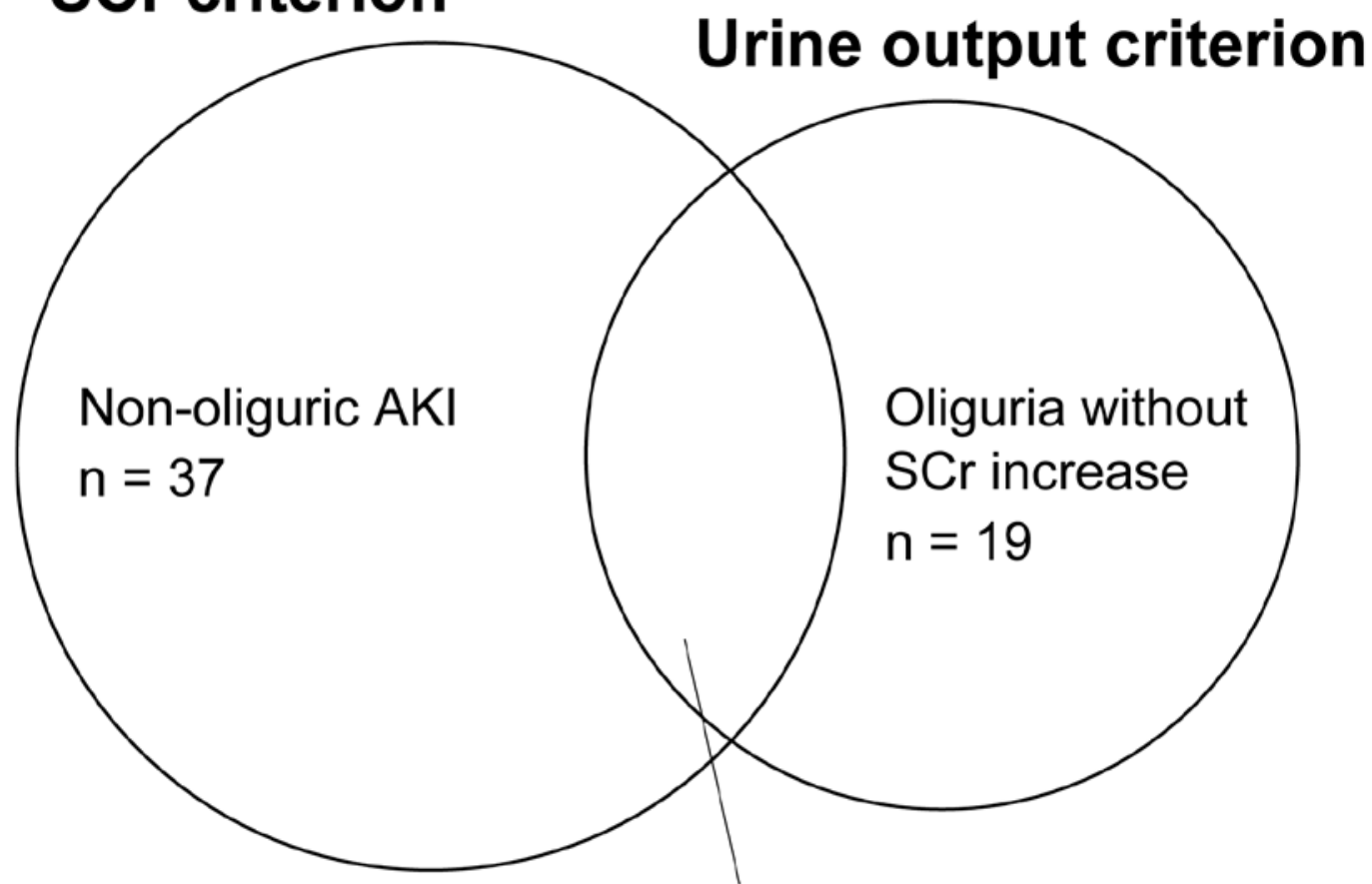

No AKI $n=84$

Oliguric AKI with SCr increase $\mathrm{n}=16$ 
Figure 2. The lengths of ICU (A) and hospital (B) stays according to AKI status. ICU, intensive care unit; AKI, acute kidney injury. 
A

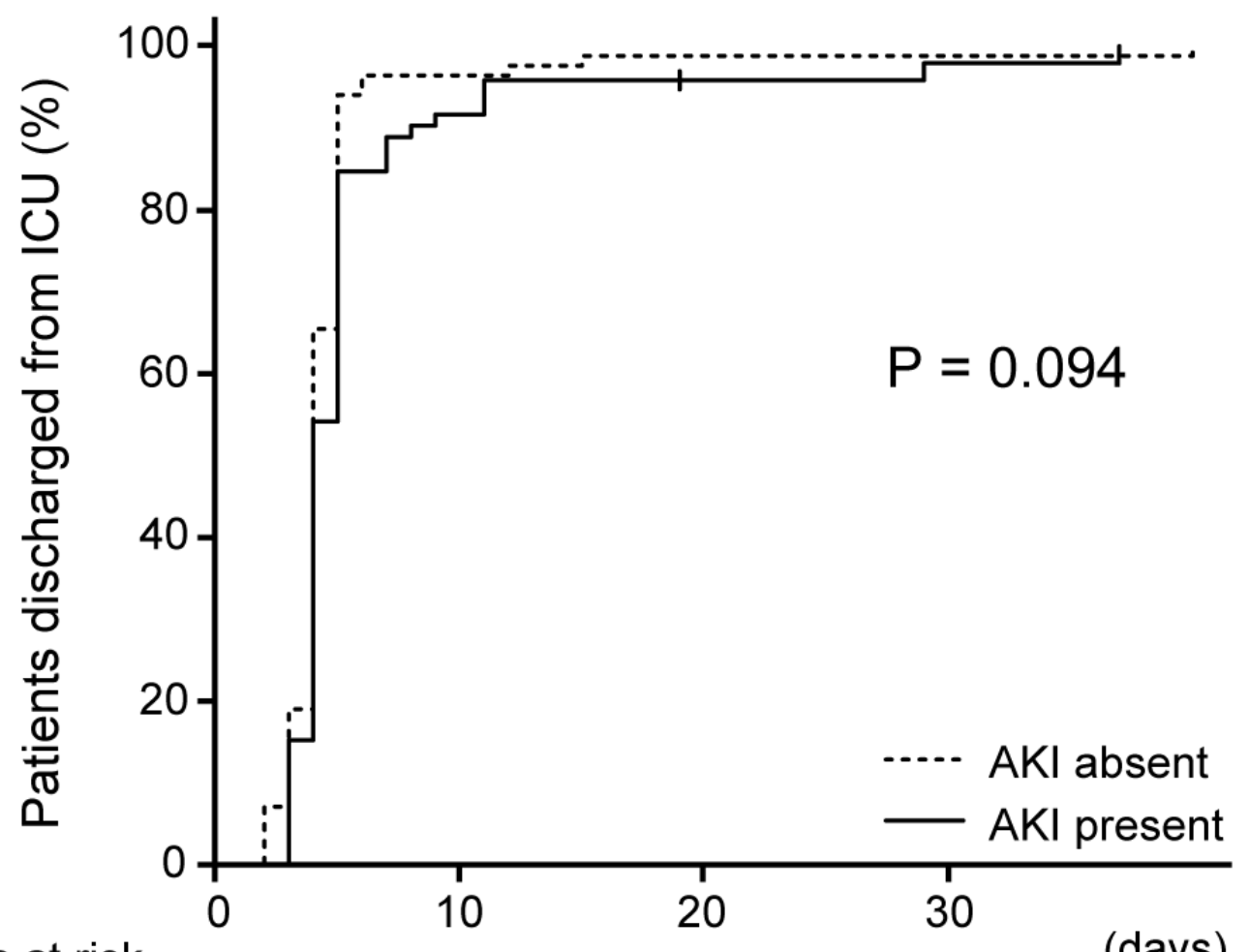

Patients at risk

AKI absent 84

AKI present 72

$\begin{array}{ll}3 & 1 \\ 6 & 2\end{array}$

1

1

B

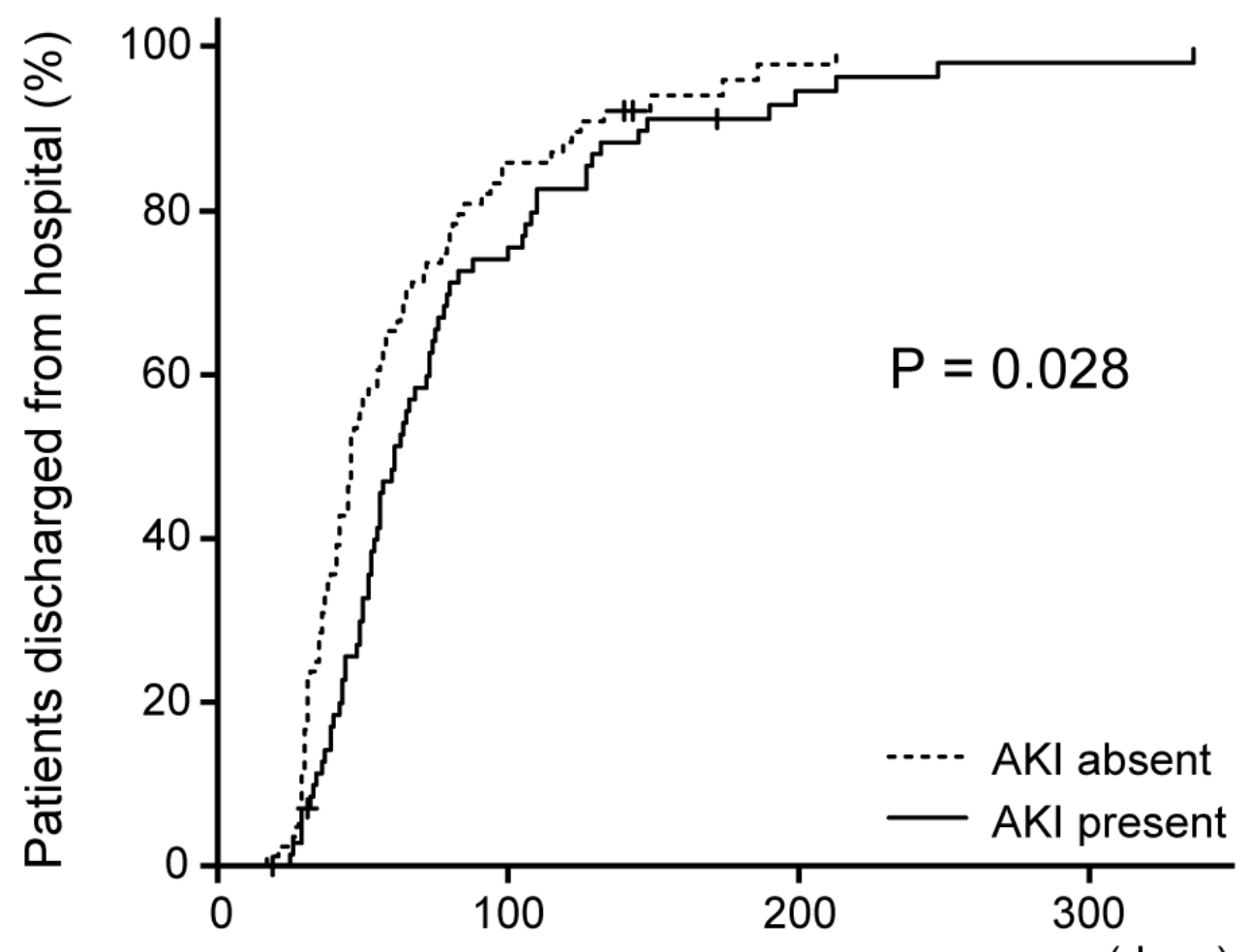

Patients at risk

AKI absent 84

AKI present 72

11

17

1

0

(days) 
Acute kidney injury after pediatric liver transplantation: incidence, risk factors, and association with outcome

Authors: Miho Hamada, et al.

\section{Supplemental data 1}

Clinical outcomes stratified by AKI stages or AKI definitions

Table 1. Clinical outcomes stratified by AKI stages

\begin{tabular}{lccccc}
\hline & $\begin{array}{c}\text { No AKI } \\
(\mathrm{n}=84)\end{array}$ & $\begin{array}{c}\text { Stage 1 AKI } \\
(\mathrm{n}=34)\end{array}$ & $\begin{array}{c}\text { Stage 2 AKI } \\
(\mathrm{n}=32)\end{array}$ & $\begin{array}{c}\text { Stage 3 AKI } \\
\text { (n=6) }\end{array}$ & P value \\
\hline Time to discharge & & & & & \\
(days) & & & & & \\
From ICU & $4(4-5)$ & $4(3-5)$ & $5(4-5)$ & $5(4-5)$ & 0.088 \\
From hospital & $46(35-78)$ & $59(43-80)$ & $64(52-106)$ & $61(44-76)$ & 0.154 \\
In-hospital mortality & $3(3.6 \%)$ & $0(0.0 \%)$ & $2(6.3 \%)$ & $1(16.6 \%)$ & 0.141 \\
\hline Data are medians (interquartile range) or numbers (percentage). AKI, acute kidney injury;
\end{tabular}

Table 2. Clinical outcomes stratified by AKI definitions

AKI-Cr and

\begin{tabular}{|c|c|c|c|}
\hline No AKI & AKI-Cr only & AKI-UO only & AKI-UO \\
\hline$n=84$ & $(\mathrm{n}=37)$ & $(\mathrm{n}=19)$ & $(\mathrm{n}=16)$ \\
\hline
\end{tabular}

Time to discharge

(days) 


$\begin{array}{cccccc}\text { From ICU } & 4(4-5) & 4(3-5) & 4(4-5) & 5(4-7) & 0.050 \\ \text { From hospital } & 46(35-78) & 63(43-79) & 50(42-105) & 65(55-110) & 0.098 \\ \text { In-hospital mortality } & 3(3.6 \%) & 2(5.4 \%) & 0(0.0 \%) & 1(6.3 \%) & 0.603\end{array}$

Data are medians (interquartile range) or numbers (percentage). AKI, acute kidney injury;

ICU, intensive care unit; AKI-Cr, acute kidney injury defined based on serum creatinine criterion; AKI-UO, acute kidney injury defined based on urine output criterion. 